To the Editors:

\title{
A hospital-based study on trends in deliberate self-harm in children and adolescents
}

(Index words: deliberate self-harm, adolescence, gender difference, paracetamol)

Deliberate self-harm (DSH) refers to self-inflicted injuries that may or may not cause death. DSH is common among adolescents, especially females [1]. It may be a risk factor for future suicide [2]. Using data from medical records, this study focuses on children and adolescents who were admitted to Teaching Hospital, Karapitiya following deliberate self-harm. Approval for the study was granted by the Ethical Review Committee of Faculty of Medicine, University of Ruhuna. We examined the BHTs of all adolescents (those under 19 years of age) admitted to the Teaching Hospital, Karapitiya with DSH during 2001, 2003, 2006 and 2007 (these years were selected based on availability and completeness of the records). Information about fatal DSH was obtained from the hospital police post. In a few cases, it could not be determined if the self-harm was deliberate; such cases were excluded.

827 admissions met the study criteria. The patients' age ranged from 9 to 18 years, with females (73\%) outnumbering males (27\%). Only 28 (3.4\%) fatalities were recorded.

Admissions of adolescents for DSH increased by 91\% from 2001 to 2007. Girls accounted for most of this increase; admissions of boys increased only slightly. This gender difference was significant ( $\mathrm{p}=0.003$ ). Admissions for DSH increased consistently with age. Only 38 children under the age of 14 years were hospitalised for DSH, compared to 257 patients who were 18 years old. Case fatality rate dropped from 8\% to 2.3\% from 2001 to 2007. Almost 99\% of the admissions involved either ingestion of a poison or an overdose of medicine. Overdoses accounted for $55 \%$ of the admissions. Ingestion of poisons (eg. washing powder, kerosene oil, agrochemicals, and poisonous plants) accounted for $43 \%$ of the admissions. The remaining cases involved burning, hanging and jumping from the roof.

Admissions after overdose of medicine increased dramatically from 2001 to 2007 (Figure 1). In 2006 and $2007,63 \%$ of the cases involved overdoses. There was a sharp gender difference in the substances ingested. About $64 \%$ of the boys used poisons and $64 \%$ of the girls had overdoses $(p<0.001)$. Admissions for paracetamol overdose increased fivefold from 2001 to 2007. In 2006-2007, 45\% of adolescents used paracetamol. BHTs recorded patients' self-reports of the quantity of tablets they had ingested. In patients whom paracetamol was the only substance ingested, the modal overdose was in the range of 20-25 tablets. The largest overdose reported was 108 tablets. None of the patients with paracetamol poisoning died.

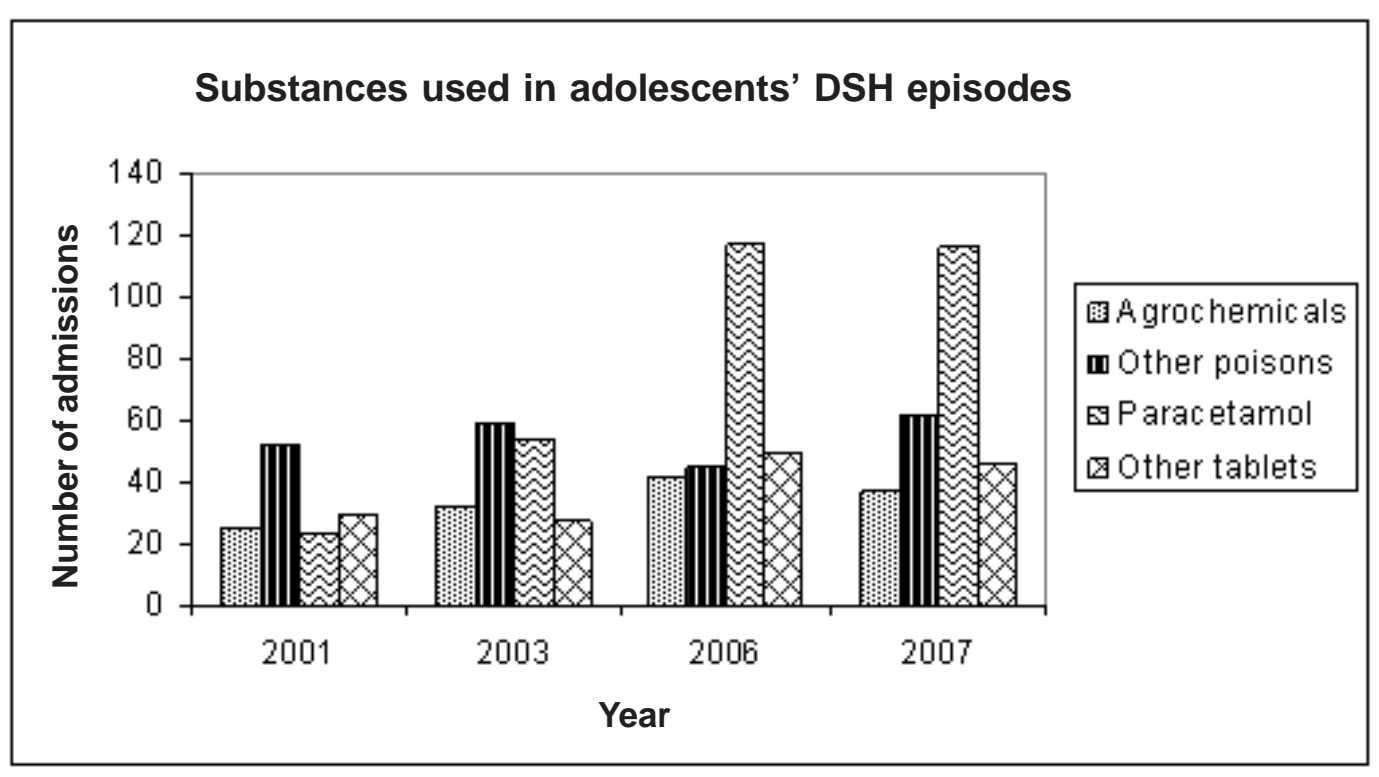

Figure 1. Substances used by adolescents for deliberate self harm. 
This study provides evidence of a recent increase in hospital admissions after DSH among children and adolescents, especially girls. This is in contrast to the decline in suicide rates reported nationally [3]. The data also suggest an increase in paracetamol overdose among adolescents, especially girls. This mirrors a broader national trend, as suggested by a recent study from the National Hospital of Sri Lanka [4]. Though there were no deaths, medical management of paracetamol overdose is costly. Furthermore it is difficult to restrict children's access to paracetamol, which is found in many households and can easily be purchased. This study has some limitations. Information on BHTs may not be accurate. Moreover, any hospital-based study of DSH underestimates its true incidence because many episodes of DSH are not admitted to a hospital.

\section{References}

1. Hawton K. Suicide and attempted suicide among children and adolescents. Beverly Hills: Sage, 1986.

2. Brent D, Perper J, Moritz G, et al. Psychiatric risk factors for adolescent suicide: A case control study. Journal of the American Academy of Child and Adolescent Psychiatry 1993; 32: 521-9.

3. Sri Lanka Police Service. Crime trends. Mode of suicide for years 2005, 2006, 2007. Available at http://www.police.lk.

4. Senaratne SMDKG, Sri Ranganathan S, Dawson AH, Buckley N, et al. Management of acute paracetamol poisoning in a tertiary care hospital. Ceylon Medical Journal 2008; 53 : 89-92.

\section{Senadheera ${ }^{1}$, J Marecek ${ }^{2}$, C Hewage ${ }^{1}$ and W A A Wijayasiri ${ }^{3}$}

${ }^{1}$ Department of Psychiatry, Faculty of Medicine, University of Ruhuna, Sri Lanka.

${ }^{2}$ Department of Psychology, Swarthmore College, USA.

${ }^{3}$ Department of Community Medicine, Faculty of Medicine, University of Ruhuna, Sri Lanka.

Correspondence: CS, e-mail <chandaniesenadheera@yahoo.co.uk>. Received 19 June and revised version accepted 1 October 2009. Competing interests: none declared. 